() Clinical Insights

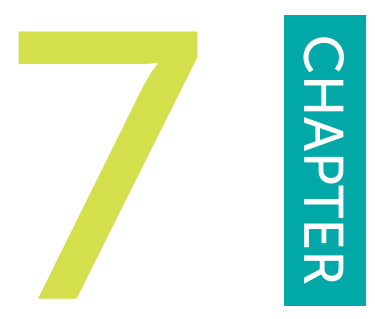

\title{
Psychological treatment for irritable bowel syndrome
}

Katleen Bogaerts \& Lukas Van Oudenhove

\section{Contents}

Patient education

Gut-directed hypnotherapy

Cognitive behavioral therapy

Biofeedback

Conclusion 


\section{About the authors}

\section{Katleen Bogaerts}

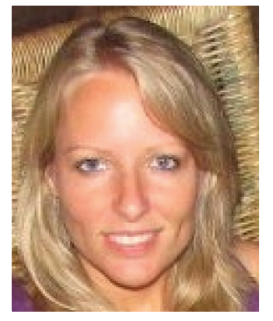

Katleen Bogaerts is a postdoctoral researcher in the Research Group on Health Psychology at KU Leuven University (Belgium). Her main research interest is in medically unexplained symptoms, or bodily symptoms for which no diagnosed organic disease can be found. She has a postgraduate diploma in cognitive behavior therapy. She is the founder and manager of Tumi Therapeutics, a center of expertise for the diagnostics and treatment of hyperventilation, stress-related disorders and medically unexplained physical symptoms. Currently she works on the OT Project 'Affective processing of interoceptive information in persons with medically unexplained symptoms' and she is also a staff member of the Center for Excellence: 'Generalization in Health and Psychopathology' where she coordinates the link between research and clinical practice at the university hospitals.

\section{Lukas Van Oudenhove}

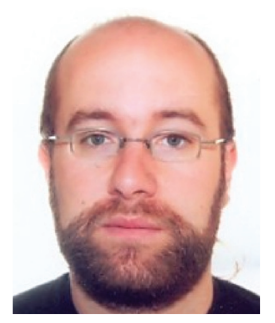

Lukas Van Oudenhove graduated as a medical doctor from KU Leuven University in 2001. After graduation he trained and worked at the Onze-Lieve-Vrouw Hospital Aalst, the University Hospitals Leuven and the Translational Research Center for Gastrointestinal Diseases of the Katholieke Universiteit Leuven. He has won several international research awards and in 2012, he was chosen as a 'Rising Star' by United European Gastroenterology. He is a member of the editorial board of the journal Neurogastroenterology \& Motility and is the co-chair of the Rome IV committee on psychosocial aspects of functional gastrointestinal (GI) disorders. He was appointed as a research professor of the KU Leuven Special Research Fund; he is currently working on a project titled 'Gut-brain interactions in visceral pain and the control of food intake'. His research on central mechanisms of GI sensitivity and psychological influences on $\mathrm{Gl}$ sensation has been internationally authoritative, as reflected by more than 60 peer-reviewed publications and numerous presentations at international scientific meetings. 


\section{Learning points}

\section{After reading this chapter you will know:}

Irritable bowel syndrome is a 'functional somatic syndrome' resulting from an interaction between biological, psychological and social factors.

Psychological factors may interfere with brain processing of visceral signals as well as with gut physiology (through the autonomic nervous system) in the generation of IBS symptoms, providing a rationale for psychological treatment.

Psychotherapy, especially cognitive-behavioral therapy is the best studied psychological treatment modality showing benefit in IBS, followed by hypnotherapy.

Patient education and various forms of biofeedback are other promising treatment options.

Further research is needed to identify the active components of these different treatment modalities as well as to overcome methodological limitations, which is needed to tailor these treatment options better to individual patients' needs.

\section{Summary}

This chapter provides an overview of psychological treatment options for irritable bowel syndrome. After briefly introducing each treatment modality, the focus lies on recent evidence supporting their efficacy. Cognitivebehavioral psychotherapy is the best-studied treatment option, followed by hypnotherapy and other promising treatments including patient (psycho) education and various forms of biofeedback. Further research is needed to overcome the limitations of current studies as well as to identify the 'active components' responsible for the efficacy of psychological treatments of IBS. 
Increasing evidence suggests that there are overlapping mechanisms underlying 'functional somatic syndromes' (Box 7.1) [1], including an autonomic imbalance and abnormal interoceptive processes in the CNS. The latter means that the brain processes and modulates bodily signals in an abnormal way, causing these signals to be perceived as (painful) physical symptoms. Importantly, both these pathophysiological processes may be influenced by psychological factors and/or psychiatric comorbidity. In irritable bowel syndrome (IBS), Naliboff et al. [2] suggest that altered autonomic balance causes altered bowel habits, stress-induced hyperalgesia results in abdominal pain/discomfort and enhanced activation of ascending arousal systems creates hypervigilance towards sensory stimuli. In addition to the physiological dysregulation, conditioned fear responses, avoidance behavior and 'unhelpful' cognitions can be important maintaining factors in the chronicity of IBS symptoms.

Based on these observations, several nonpharmacological interventions involving changes in behavior and/or

\section{Box 7.1. Heart rate variability.}

- Heart rate variability (HRV) is a measure of interbeat interval fluctuations in heart rate and is emerging as a possible descriptor of the brain functional organization contributing to homeostasis.

- HRV has been considered to be an inferential index of prefrontal inhibitory capacity and is shown to be linked to cognitive performance [37,38]. Commonly used indices are frequency domain and time domain measures. Three components can be identified in the frequency domain: a very low frequency component (VLF) that corresponds to the power below 0.04 (or 0.05 ) $\mathrm{Hz}$, a low-frequency (LF) component centered around $0.1 \mathrm{~Hz}$, and a high frequency (HF) component that corresponds to the (normal) respiratory frequency band $(0.15-0.4 \mathrm{~Hz})$.

- Efferent vagal (parasympathetic) activity is a major contributor to the HF component. In addition, respiratory sinus arrhythmia (RSA) is referred to as the naturally occurring variation in heart rate that occurs during a breathing cycle and is also a measure of parasympathetic nervous system activity. Lower resting HRV values are found in patients with functional syndromes [39].

- The midbrain periaqueductal gray area mediates pain modulation through the autonomic nervous system. The ventral portion increases parasympathetic activity and reduces the response to pain.

- Pereira and colleagues showed that HF power significantly increased and the LF/HF power ratio decreased following stimulation of the ventral portion in patients with chronic or unexplained pain [40]. In addition, the severity of abdominal pain was negatively related to $\mathrm{HF}$ power and positively related to the LF/HF ratio in constipation-predominant IBS patients [41]. 
Functional somatic syndromes: irritable bowel syndrome (IBS) can be conceptualized as one of many so-called 'functional somatic syndromes' (FSS), including fibromyalgia, chronic fatigue syndrome, multiple chemical sensitivity, non-cardiac chest pain, (Gulf) war syndrome, chronic hyperventilation syndrome, and many others. To date, there are no confirmed biomarkers that are distinctive of different FSS. On the other hand, there is substantial overlap in case definitions for different FSS and the patients identified by those diagnoses overlap considerably. Another reason for arguing that FSS are closely related is the fact that they share many non-symptom characteristics, such as female predominance, substantial correlations with negative affectivity, psychiatric comorbidity (e.g., anxiety and depression), impairments in functional status or quality of life, excessive healthcare use, response to the same nonpharmacological therapies, and others [1].

Irritable bowel syndrome: a very common functional digestive disorder characterized by abdominal pain or discomfort (including bloating) and disturbed defecation with no clear biochemical or structural abnormalities.

cognition have been studied for patients with IBS. It has been proposed that these interventions may impact on stress-related brain-gut interactions and interfere with cortical brain circuits involved in pain modulation [3]. Examples of these interventions include patient education, hypnotherapy, cognitive behavioral therapy (CBT), relaxation and biofeedback training. As relaxation training is often included as a component of other interventions (such as CBT or biofeedback) and has also been used as a control condition for other psychological treatments, it will not be described separately.

\section{Patient education}

Patients with IBS have to cope not only with their symptoms but also with the social stigma that is still attached to functional somatic syndromes. The diffuse symptomatology, unclear etiology and lack of medical diagnosis and clear-cut treatment strategy may frustrate doctors when dealing with these patients, who are frequently considered to be 'difficult' and 'timeconsuming'. IBS patients have often felt misunderstood because physicians have not taken their problems seriously. Therefore, a strong demand exists for more information and instruction about their disorder [4].

Bengtsson and colleagues found that educating women with IBS on medical care, physical activity, stress management (SM), diet and health insurance led to significant improvements in vitality and abdominal pain as well as to a reduced number of healthcare visits [5]. These results 


Cognitive behavioral therapy (CBT): based on the learning
principles of classical and operant conditioning as well as on insights
in cognitive research. CBT is most closely allied with the scientist-
practitioner model in which clinical practice and research support
each other, with an emphasis on a clear operationalization of the
problem, measuring changes in cognition and behavior and the attainment of
goals. CBT is based on the idea that the autonomic arousal caused by 'unhelpful'
thoughts and avoidance behavior may trigger the gastrointestinal symptoms,
causing vicious circles. CBT interventions include education about IBS symptoms
and their relationship to stress, self-monitoring of IBS symptoms, relaxation skills,
restructuring of cognitions, attentional control skills, and (interoceptive) exposure.
CBT is usually applied in the form of weekly 1-h sessions over a period of
$8-12$ weeks.
Biofeedback: a method in which physiological signals are measured and viewed
'online' by the therapist and patient by use of an instantaneous electronic display.
The effect of a catastrophizing thought, attention, posture, way of breathing, and
so on is thereby immediately visible. The goal is that the patient learns to recog-
nize his own bodily signals (awareness) and to influence them by use of oper-
ant conditioning principles (reinforcement of desired effects), first by use of the
biofeedback equipment, and later without (process of internalization). Ultimately,
the patient should be able to integrate and generalize the biofeedback modulated
skills into his daily life.

call for an implementation of patient education as part of a multicomponent treatment program. Other research has confirmed the effectiveness of psychoeducation group treatments relative to usual care in IBS $[6,7]$.

\section{Gut-directed hypnotherapy}

In a landmark study published in the Lancet in 1984, Whorwell and colleagues first demonstrated the superior efficacy of hypnotherapy compared with psychotherapy and placebo on core IBS symptoms (abdominal pain, bowel habit) and general wellbeing in 30 refractory patients [8]. Another Lancet publication in 1989 demonstrated that group hypnotherapy is as effective as individual treatment [9]. Since then, numerous other studies have been published and the topic has been the subject of several systematic reviews and meta-analyses, including a Cochrane review [10], with the most recent one being published in 2009 [11] Although all these reviews emphasize important methodological limitations of most of the published studies (small sample size, lack of adequate control groups and interventions, lack of standardization of outcomes), the uniform conclusion is that the 


$\begin{aligned} & \text { Systematic review: an unbiased synthesis of the available research } \\ & \text { about a specific topic. Systematic reviews use a methodology for a } \\ & \text { comprehensive search of the literature for relevant studies based on } \\ & \text { a priori search terms or criteria. Thus, a bias-free list of citations is } \\ & \text { developed and those that do not fulfill the established criteria are }\end{aligned}$
not included.
Gut-directed hypnotherapy (GDH): was first developed in Manchester (UK) in
the early 1980s and teaches the patients the necessary hypnotic skills to control
and help normalize gut function and symptoms. After an initial intake session de-
voted to history, reassurance about hypnosis and psychoeducation on gut func-
tion and gut-brain interactions, the patient attends up to 12 sessions of hypnotic
induction and suggestions over a 3-month period. Hypnotic induction is achieved
by the usual means, such as progressive relaxation and the suggestion of going
to a special place. Gradually, more specific suggestions aimed at controlling and
normalizing gut function are introduced.

therapeutic effect of hypnotherapy on primary IBS symptoms is superior to waiting list control and usual medical management and that the effect appears to be long-lasting. A recent large Swedish randomized controlled trial demonstrated higher effectiveness of gut-directed hypnotherapy (GDH) compared with supportive psychotherapy and waiting list control on core IBS symptoms in refractory patients, an effect that was sustained up to 1 year [12]. Therapeutic gains have been reported to be maintained up to 5 years after treatment [13,14]. Whorwell and colleagues demonstrated that positive mood, high alexithymia and presence of comorbid anxiety were predictors of a good response to hypnotherapy [15] . A recent high-quality large Austrian trial demonstrated efficacy of group GDH compared with supportive talks with medical treatment on IBS-related quality of life as well as psychological symptoms [16]. Furthermore, three randomized controlled trials demonstrating efficacy of GDH for IBS or functional abdominal pain have been published, as systematically reviewed in [17].

Despite this growing body of evidence for the efficacy of GDH as a treatment for IBS, the mechanisms of action of hypnotherapy remain elusive. A recent study did not find any effect on gastrointestinal motility parameters, including gastric emptying and small bowel and colonic transit time [14]. Vlieger et al. demonstrated that hypnotherapy does not have a significant effect on rectal sensitivity in children [18], whereas a normalization of rectal sensitivity after GDH has been shown in some, but not all, studies in 
adults [11]. A study in adult patients showed that hypnotherapy reduces the sensory and motor component of the gastrocolonic response, which is exaggerated in IBS patients [19]. The vast majority of studies have shown an effect on stress, anxiety, depression and somatization, and some of these studies demonstrated an association between psychological improvement and improvement of core IBS symptoms [11]. Finally, there is rather limited evidence that GDH may influence autonomic nervous system activity [11].

In summary, there is growing evidence for the efficacy of GDH in IBS, although more high-quality trials, as have been published recently, are needed.

\section{Cognitive behavioral therapy}

CBT has been studied the most extensively of all psychological interventions and has been reported to be effective in the long term in individual and group treatment of IBS when compared with no treatment, standard medical care, placebo or active psychological interventions such as psychoeducation, supportive therapy or SM/stress reduction treatment [20-22]. A review and meta-analysis by Ford et al. showed that - from a range of different psychological therapies that were able to significantly reduce physical symptoms in patients with IBS - studies on CBT provided the strongest evidence, although it needs to be mentioned that this study only included two hypnotherapy studies that were not of the highest quality [20].

In a recent study, symptoms and disability caused by IBS were significantly more reduced at post-test level when using a combination of CBT and medical treatment compared with medical treatment alone [23]. Drossman and colleagues reported significantly greater improvements after 12 weeks CBT compared with education alone in women with moderate-to-severe functional bowel disorders [24]. However, in a rigorously conducted randomized-controlled trial the hypothesis that CBT would be superior to relaxation and standard care alone in IBS patients could not be confirmed [25], as patients in all three treatment groups improved significantly in terms of self-reported bowel symptoms. At the start of this study, patients within each experimental condition were provided with a detailed explanation of a biopsychosocial model of IBS as well as basic education about gut function coupled with dietary advice. A possible explanation for the results of this study could be that patient education might have been a critical component in the positive effects, for example by reducing uncertainty and fear for severe disease, as well as increasing self-efficacy.

Craske and colleagues ascribed 110 IBS patients to one of three 
experimental conditions, receiving ten sessions each: $\mathrm{SM}$; an attention control, consisting of self-monitoring of IBS symptoms, reading educational material and discussing it with the therapist (attention by therapist [AT]); and CBT with interoceptive exposure (IE) to visceral sensations [26]. The IE treatment was based on a fear of visceral sensations conceptualization of IBS symptoms. IE narrows the focus of treatment to interoceptive (visceral) cues associated with the target symptoms, unlike SM, which attempts to alter coping responses to a broad range of exteroceptive (psychosocial) life stressors. They found no differences in outcome between SM and AT, while IE significantly outperformed AT on several outcome indices, including a measure of symptom severity as well as anxiety about symptoms and overall body vigilance, and outperformed SM in some domains, including symptom severity. The results highlight the importance of directly targeting fear and avoidance of visceral sensations using repeated interoceptive exposure.

Recently, studies have been conducted investigating CBT for IBS where patients had online therapist contact via the internet (ICBT). This is a promising cost-effective treatment modality as it can be offered to IBS patients on a much larger scale than conventional psychological treatments [27].
Another promising intervention mode - at least from a theoretical point of view - is acceptance and commitment therapy (ACT). ACT is a so-called 'third generation' behavioral therapy based on the relational frame theory [28]. Some forms of CBT focus mainly on behavioral avoidance, sometimes neglecting experiential avoidance (i.e., avoiding all inner experiences such as aversive thoughts, feelings and somatic discomfort). Many individuals with IBS engage in a variety of experiential avoidance strategies, such as distraction or suppression of thoughts, feelings and bodily sensations. We believe that ACT may show merit as a treatment modality for IBS as a prime focus is given to experiential avoidance through interoceptive exposure. Specifically, ACT encourages interoceptive exposure while monitoring (i.e., observation and labeling) one's thoughts, feelings and bodily sensations in a neutral, detached manner. Unlike cognitive therapy, which seeks to challenge the irrationality of thoughts, in ACT the content of the thought is not so relevant as its functionality (is it helpful for you or not?). The main goal of ACT is to increase psychological and physiological flexibility, by incorporating techniques of mindfulness (living in the present moment and engaging fully in what one is doing rather than 'getting lost' in ones thoughts), acceptance (allowing ones feelings, thoughts, 
and sensations to be as they are, letting them come and go rather than trying to control them), metaphors, experiential exercises, value-guided behavioral interventions, and cognitive defusion. As such, IBS patients can learn how to stop 'fighting' their symptoms, but rather to perceive them as harmless - even if uncomfortable transient inner events. Although ACT has been proven to be effective as a treatment for several disorders, direct studies of the efficacy of ACT for IBS do not yet exist.

\section{Biofeedback}

\section{Colonic motility}

The first attempts to treat IBS with biofeedback focused on modifying colonic motility. Furman tried to teach five patients with diarrheapredominant IBS to alternatively increase and decrease bowel sounds by use of an electronic stethoscope [29]. All patients reported improvement in symptoms, which was said to be correlated with learning control over bowel sounds. However, other researchers could not replicate these results [30]. Therefore, the efficacy of colon-motility oriented biofeedback as a treatment for IBS is questionable.

\section{Biofeedback relaxation training}

Relaxation training implemented through biofeedback has also been studied in patients with IBS. Blanchard and colleagues repeatedly found a multicomponent biofeedback training protocol (progressive muscle relaxation, thermal biofeedback, cognitive therapy and education) to be more effective than a waiting list condition [31,32]. However, when multicomponent treatment was compared with an attention-placebo condition (pseudomeditation and electroencephalogram $\alpha$-suppression biofeedback), it was no more effective than placebo [33], suggesting that wait-list control groups do not adequately control for expectancy (placebo).

\section{Autonomic nervous system}

Several researchers have established that dysregulation of the autonomic nervous system is a component of IBS [34,35]. Given this autonomic imbalance in IBS patients, biofeedback training has also been used to decrease body muscle tension and sympathetic activity. Leahy et al. developed a computerized biofeedback game that relates variations in stress level to animated computer graphics, by monitoring electrodermal activity [36]. Symptom scores in 40 IBS patients were improved using this device, for approximately half of them over a period of 3 months. However, the fact that there was no control group nor long-term follow-up was an important limitation. 
Heart rate variability (HRV) biofeedback is designed specifically to target autonomic reactivity, primarily influencing the parasympathetic system and producing major increases in gain in the heart rate baroreflex closed loop (Box 7.1) [37-41]. When respiratory sinus arrhythmia is put in phase with respiration, baroreflexes are triggered and efficacy of respiratory gas exchange is increased.

Also, HRV biofeedback benefits from being noninvasive and having a high benefit-cost ratio. Preliminary results have shown that HRV biofeedback may have important effects on autonomic symptoms and on quality of life and pain in fibromyalgia [42]. Humphreys and Gevirtz also showed that in a sample of children with functional abdominal pain, the symptoms were improved after HRV biofeedback and a change in vagal tone was suggested as the potential mediator for this improvement [43]. In general, HRV biofeedback is a rather new, yet promising treatment tool.

\section{Electromyography}

Clinical applications of electromyographic (EMG)-based biofeedback therapy supported by randomized, controlled trials (RCTs) are limited. Patients can be taught to improve their capacity to voluntarily contract the external anal sphincter during rectal filling, either by improving the strength of the anal sphincter and improving squeeze duration (motor skills training), increasing the ability to perceive mild rectal sensations (sensory discrimination training), or combining both mechanisms (coordination training). For fecal incontinence, RCTs suggest that biofeedback combining strength training and sensory discrimination training is effective in approximately $75 \%$ of patients and is more effective than placebo. For dyssynergic defecation, RCTs show that biofeedback combining pelvic floor muscle relaxation training, practice in defecating a water-filled balloon, and instruction in effective straining is efficient in approximately $70 \%$ of patients who have failed to respond to laxative treatment. For both incontinence and dyssynergic defecation, the benefits of biofeedback last at least 12 months. A meta-analytic review of biofeedback treatment of constipation examined seven RCTs, comparing biofeedback with non-biofeedback modalities (e.g., laxatives, conservative management; $\mathrm{n}=3$ ) or comparing various biofeedback treatments, such as EMG versus balloon biofeedback ( $n=4$; [44]). Biofeedback produced a sixfold improvement in constipation symptoms compared with nonbiofeedback treatments. Furthermore, EMG biofeedback produced an almost sevenfold improvement compared with non-EMG biofeedback. 
There are, however, important limitations to all aforementioned EMG studies. Only a few RCTs used an adequate control group, such as sham biofeedback. Several studies suffered from problems in standardization of outcome measures and doubleblinding. In future research more solid research protocols using a well-designed control treatment are required to test whether biofeedback has a unique effect over and above nonspecific factors such as support, attention, expectancy to benefit from the training, the use of cognitive and emotion-regulation techniques and relaxation. In addition, simply the use of technologically impressive equipment and the presentation of a clear and plausible explanation for the complaints might improve self-efficacy and cause the larger effect.

One exception is the recent study of Hart et al., in which the efficacy of anorectal biofeedback (AB; learning to isolate the anal sphincter using an EMG probe) for constipation was compared with a biofeedback control (learning to relax trapezius or temporalis muscles with EMG feedback) treatment [45]. They further investigated the extent to which self-reported childhood sexual/ physical abuse predicted biofeedback outcome. Biofeedback therapy targeting the trapezius muscle has been found to alleviate temporomandibular joint pain, tension headache, chronic orofacial pain and chronic back pain, but does not appear to benefit pelvic floor relaxation and bowel function and has an effect only on the targeted muscle group [46]. Consequently, it is a suitable control for the 'nonspecific' effects of $A B$ (e.g., establishment of a therapeutic relationship, instruction in relaxation of a muscle group and attending sessions at a medical center). Study results showed that only $A B$ produced clinical improvements in constipation severity and quality of life. The data also suggest that a history of childhood sexual and physical abuse may be associated with diminished improvement after biofeedback for pain severity and impaired mental quality of life. Unfortunately, a longer term follow-up period is lacking in this study. A recent study of Patcharatrakul and Gonlachanvit showed that the presence of IBS in dyssynergic defecation did not affect the outcome of biofeedback therapy [47]. In addition, treating dyssynergic defecation patients with IBS by biofeedback therapy improved both constipation and other IBS symptoms.

\section{The future of biofeedback therapy}

Overall, the future of biofeedback therapy depends on improving research methodology. RCTs on dyssynergic defecation and fecal incontinence have shown that studies with adequate study samples, standardized treatment protocols and outcomes, 
and adequate length of follow-up yield more consistent results. There are many reasons why biofeedback may be an interesting therapeutic tool in IBS. First of all, it helps making the invisible visible. Physiological monitoring can help to uncover abnormal physiological patterns (i.e., autonomic imbalance) and to evaluate changes that occur as a result of treatment interventions. Secondly, biofeedback therapy might be more appealing to patients with IBS than traditional psychotherapy. These patients often have the feeling that their physicians see their symptoms as 'all in the mind'. Such questioning of the legitimacy of the symptoms puts the patients in the position of having to prove that they are ill. This often leads to dualistic thinking on behalf of the patient, wanting to prove that the problem is all in the body, not the mind. Biofeedback specifically targets physiological processes that are thought to be related to the patient's complaint. In this way, biofeedback can become a 'Trojan horse' technique for helping such patients become aware of and deal with the emotional issues that may produce the physical complaints. It could therefore generate increased treatment acceptance and adherence as well as a larger sense of self-efficacy [48].

\section{Conclusion}

To date CBT remains the most effective psychological treatment for IBS, based on the outcome and number of sound RCTs compared with other modalities.

Although the majority of the studies mentioned in this chapter have reported a positive effect of the psychological interventions studied, a lot of them suffer from substantial methodological limitations. Further research should deal with issues such as adequate blinding of conditions, use of validated outcome measures, larger sample sizes, a definition of IBS according to standardized criteria and long-term follow-ups. Moreover, to date, it is not yet clear whether psychological treatments are more effective than psychotropic agents in the management of IBS, although some study results show the superiority of psychological treatments over antidepressants in terms of longterm reduction in healthcare costs [49]. Studies comparing both treatment modalities of IBS are needed urgently.

Furthermore, an analysis of the critical underlying mechanisms causing beneficial changes in attentional, cognitive and emotional factors, as well as in interoceptive accuracy of gastrointestinal sensations is needed. Also, disentangling the effects of different components in multicomponent approaches would be interesting. For example, biofeedback is based on the same learning principles as CBT. Therefore, combining both might be a promising 
treatment strategy for IBS, begging further research. Future research should also focus on the refinement of treatment packages in function of patient characteristics, symptom severity and illness duration.

Adding education about the biopsychosocial model of IBS to standard medical care can enhance treatment effects. In our opinion, further psychological treatment for IBS should be recommended for patients: for whom standard medical care does not sufficiently work after a few months; for those reporting several other physical symptoms besides the typical IBS related ones (e.g., high symptom reporters); for patients with problematic coping skills; and for patients with a history of physical, sexual or emotional abuse/neglect and/or psychiatric co-morbidity. A combination of medical management, CBT, hypnosis and biofeedback might be an interesting approach, paying attention to specific patient characteristics such

\section{References}

1. Wessely S, Nimnuan C, Sharpe M. Functional somatic syndromes: one or many? Lancet 354, 936-939 (1999).

2. Naliboff BD, Fresé MP, Rapgay L. Mind/ body psychological treatments for irritable bowel syndrome. Evid. Based Complement. Alternat. Med. 5, 41-50 (2008).

3. Lackner JM, Lou Coad M, Mertz HR et al. Cognitive therapy for irritable bowel syndrome is associated with reduced limbic activity, Gl symptoms, as hypnotizability, personality, trauma, presence of maintaining factors such as catastrophizing, avoidance, hypervigilance towards symptoms, and so on. A good referral therefore requires sound diagnostical work (e.g., medical tests, psychophysiological measures, questionnaires and interview) as well as adequate doctor-patient communication.

\section{Financial \& competing interests disclosure}

The authors have no relevant affiliations or financial involvement with any organization or entity with a financial interest in or financial conflict with the subject matter or materials discussed in the manuscript. This includes employment, consultancies, honoraria, stock ownership or options, expert testimony, grants or patents received or pending, or royalties.

No writing assistance was utilized in the production of this manuscript.

and anxiety. Behav. Res. Ther. 44, 621-638 (2006).

4. Dancey CP, Backhouse S. Towards a better understanding of patients with irritable bowel syndrome. J. Adv. Nurs. 18, 1443-1450 (1993).

5. Bengtsson M, Ulander K, Bergh Börgdal E, Christensson A, Ohlsson B. A course of instruction for women with irritable bowel syndrome. Patient Educ. Couns. 62, 118-125 (2006). 
6. Colwell LJ, Prather CM, Phillips SF, Zinsmeister AR. Effects of an irritable bowel syndrome educational class on health-promoting behaviors and symptoms. Am. J. Gastroenterol. 93, 901-905 (1998).

7. Saito YA, Prather CM, Van Dyke $C T$, Fett S, Lock GR 3rd. Effects of multidisciplinary education on outcomes in patients with irritable bowel syndrome. Clin. Gastroenterol. Hepatol. 2, 576-584 (2004).

8. Whorwell PJ, Prior A, Faragher EB. Controlled trial of hypnotherapy in the treatment of severe refractory irritable bowel syndrome. Lancet 2, 1232-1234 (1984).

9. Harvey RF, Hinton RA, Gunary RM, Barry RE. Individual and group hypnotherapy in treatment of refractory irritable bowel syndrome. Lancet 1, 424-425 (1989).

10. Webb AN, Kukuruzovic RH, CattoSmith AG, Sawyer SM. Hypnotherapy for treatment of irritable bowel syndrome. Cochrane Database Syst. Rev. 4, CD005110 (2007).

11. Hefner J, Rilk A, Herbert BM, Zipfel S, Enck P, Martens U. Hypnotherapy for irritable bowel syndrome - a systematic review. Z. Gastroenterol. 47, 1153-1159 (2009).

12. Lindfors $P$, Unge $P$, Arvidsson $P$ et al. Effects of gut-directed hypnotherapy on IBS in different clinical settings - results from two randomized, controlled trials. Am. J. Gastroenterol. 107, 276-285 (2012).

13. Gonsalkorale WM, Miller $V$, Afzal A et al. Long term benefits of hypnotherapy for irritable bowel syndrome. Gut 52, 1623-1629 (2003).

14. Lindfors $\mathrm{P}$, Unge $\mathrm{P}$, Nyhlin $\mathrm{H}$ et al. Long-term effects of hypnotherapy in patients with refractory irritable bowel syndrome. Scand. J. Gastroenterol. 47, 414-420 (2012).
15. Carruthers HR, Morris J, Tarrier N, Whorwell PJ. Mood color choice helps to predict response to hypnotherapy in patients with irritable bowel syndrome. BMC Complement. Altern. Med. 10, 75 (2010).

16. Moser G, Trägner S, Gajowniczek EE et al. Long-term success of GUT-directed group hypnosis for patients with refractory irritable bowel syndrome: a randomized controlled trial. Am. J. Gastroenterol. 108, 602-609 (2013).

17. Rutten JM, Reitsma JB, Vlieger AM, Benninga MA. Gut-directed hypnotherapy for functional abdominal pain or irritable bowel syndrome in children: a systematic review. Arch. Dis. Child. 98, 252-257 (2013).

18. Vlieger AM, Van den Berg MM, Menko-Frankenhuis C, Bongers ME, Tromp E, Benninga MA. No change in rectal sensitivity after gut-directed hypnotherapy in children with functional abdominal pain or irritable bowel syndrome. Am. J. Gastroenterol. 105, 213-218 (2010).

19. Simrén $M$, Ringström $G$, Björnsson ES, Abrahamsson H. Treatment with hypnotherapy reduces the sensory and motor component of the gastrocolonic response in irritable bowel syndrome. Psychosom. Med. 66, 233-238 (2004).

20. Ford AC, Talley NJ, Schoenfeld PSE, Quigley MM, Moayyedi P. Efficacy of antidepressants and psychological therapies in irritable bowel syndrome: systematic review and meta-analysis. Gut 58, 367-378 (2009).

21. Moss-Morris R, McAlpine L, Didsbury $L P$ et al. A randomized controlled trial of a cognitive behavioural therapybased self management intervention for irritable bowel syndrome in primary care. Psychol. Med. 40, 85-94 (2010).

22. Palsson OS, Whitehead WE. Psychological treatments in functional gastrointestinal disorders: a primer 
for the gastroenterologist. Clin. Gastroenterol. Hepatol. 11, 208-216 (2013).

23. Mahvi-Shirazi M, Fathi-Ashtiani A, Rasoolzade-Tabatabaei SK, Amini M. Irritable bowel syndrome treatment: cognitive behavioral therapy versus medical treatment. Arch. Med. Sci. 8, 123-129 (2012).

24. Drossman DA, Toner BB, Whitehead WE et al. Cognitive-behavioral therapy versus education and desipramine versus placebo for moderate to severe functional bowel disorders. Gastroenterology 125, 19-31 (2003).

25. Boyce P, Talley NJ, Balaam B, Koloski NA, Truman G. A randomized controlled trial of cognitive behavior therapy, relaxation training, and routine clinical care for the irritable bowel syndrome. Am. J. Gastroenterol. 98, 2209-2218 (2003).

26. Craske MG, Wolitzky-Taylor KB, Labus J et al. A cognitive-behavioral treatment for irritable bowel syndrome using interoceptive exposure to visceral sensations. Behav. Res. Ther. 49, 413-421 (2011).

27. Ljottson B, Andersson G, Andersson E et al. Acceptability, effectiveness, and cost-effectiveness of internetbased exposure treatment for irritable bowel syndrome in a clinical sample: a randomized controlled trial. $B M C$ Gastroenterol. 11, 1-13 (2011).

28. Hayes SC, Luoma JB, Bond FW, Masuda A, Lillis J. Acceptance and commitment therapy: model, processes and outcomes. Behav. Res. Ther. 44, 1-25 (2006).

29. Furman S. Intestinal biofeedback in functional diarrhea: a preliminary report. J. Behav. Ther. Exp. Psychiatry 4, 317-321 (1973).

30. Radniz CL, Blanchard EB. Bowel sounds biofeedback as a treatment for irritable bowel syndrome. Biofeedback Self Regul. 13, 169-179 (1983).
31. Neff DF, Blanchard EB. A multicomponent treatment for the irritable bowel syndrome. Behav. Ther. 18, 70-73 (1987).

32. Schwarz SP, Blanchard EB, Neff DF. Behavioral treatment of irritable bowel syndrome: a 1 year follow-up study. Biofeedback Self Regul. 11, 189-198 (1986).

33. Blanchard EB, Schwarz SP, Suls JM et al. Two controlled evaluations of multicomponent psychological treatment of irritable bowel syndrome. Behav. Res. Ther. 30(2), 175-189 (1992).

34. Aggarwal A, Cutts TF, Abell TL et al. Predominant symptoms in irritable bowel syndrome correlate with specific autonomic nervous system abnormalities. Gastroenterology 106, 945-950 (1994).

35. Smart $\mathrm{HL}$, Atkinson M. Abnormal vagal function in irritable bowel syndrome. Lancet 2(8557), 475-478 (1987).

36. Leahy A, Clayman C, Mason I, Lloyd G, Epstein O. Computerised biofeedback games: a new method for teaching stress management and its use in irritable bowel syndrome. J. R. Coll. Physicians Lond. 32, 552-556 (1998).

37. Duschek S, Muckenthaler M, Werner $\mathrm{N}$, del Paso GA. Relationships between features of autonomic cardiovascular control and cognitive performance. Biol. Psychol. 8, 110-117 (2009).

38. Thayer JF, Hansen AL, Saus-Rose E, Johnsen $\mathrm{BH}$. Heart rate variability, prefrontal neural function, and cognitive performance: the neurovisceral integration perspective on selfregulation, adaptation, and health. Ann. Behav. Med. 37, 141-153 (2009).

39. Tak LM, Riese $H$, de Bock $G H$, Manoharan A, Kok IC, Rosmalen JG. As good as it gets? A meta-analysis and systematic review of methodological quality of heart rate variability studies in functional somatic disorders. Biol. Psychol. 82, 101-110 (2009). 
40. Pereira EA, Lu G, Wang S et al. Ventral periaqueductal grey stimulation alters heart rate variability in humans with chronic pain. Exp. Neurol. 223, 574-581 (2010).

41. Cain KC, Jarrett ME, Burr RL, Hertig VL, Heitkemper MM. Heart rate variability is related to pain severity and predominant bowel pattern in women with irritable bowel syndrome. Neurogastroenterol. Motil. 19, 110-118 (2007).

42. Hassett AL, Radvanski DC, Vaschillo EG et al. A pilot study of the efficacy of heart rate variability (HRV) biofeedback in patients with fibromyalgia. Appl. Psychophysiol. Biofeedback 32, 1-10 (2007).

43. Humphreys P, Gevirtz RN. Treatment of recurrent abdominal pain: components analysis of four treatment protocols. J. Pediatr. Gastroenterol. Nutr. 31, 47-51 (2000).

44. Koh CE, Young CJ, Young JM, Solomon MJ. Systematic review of randomized controlled trials of the effectiveness of biofeedback for pelvic floor dysfunction. Br. J. Surg. 95, 1079-1087 (2008).
45. Hart SL, Lee JW, Berian J, Patterson TR, Del Rosario A, Varma MG. A randomized controlled trial of anorectal biofeedback for constipation. Int. J. Colorectal Dis. 27, 459-466 (2012).

46. Wang J, Luo MH, Qi QH, Dong ZL. Prospective study of biofeedback retraining in patients with chronic idiopathic functional constipation. World J. Gastroenterol. 9, 2109-2113 (2003).

47. Patcharatrakul T, Gonlachanvit S. Outcome of biofeedback therapy in dyssynergic defecation patients with and without irritable bowel syndrome. J. Clin. Gastroenterol. 45, 593-598 (2011).

48. Katsamanis M, Lehrer PM, Escobar JI, Gara MA, Kotay A, Liu R. Psychophysiologic treatment for patients with medically unexplained symptoms: a randomized controlled trial. Psychosomatics 52, 218-229 (2011).

49. Creed F, Fernandes L, Guthrie E et al. The costeffectiveness of psychotherapy and paroxetine for severe irritable bowel syndrome. Gastroenterology 124, 303-317 (2003). 


\section{Multiple choice questions}

Which psychological treatment option for irritable bowel syndrome (IBS) is supported by the best evidence?

a. Hypnotherapy

b. Stress management

c. Biofeedback

d. Cognitive-behavioral psychotherapy

2. Which physiological parameter has not been studied as a potential target for biofeedback therapy?

Autonomic nervous system function, for example, heart rate variability

Electromyography

Stress hormone system function

Colonic motility

3. Which key component of cognitive-behavioral psychotherapy might be crucial in the treatment of IBS?

Behavioral activation

Interoceptive exposure

Challenging the irrationality of thoughts

Stress management

What is not typical for a functional somatic syndrome?

Male predominance

The presence of an autonomic imbalance and abnormal interoceptive processes in the CNS

c. Difficult doctor-patient relationship

d. Positive response to psychological treatment 